\title{
A Kullback-Leibler Approach to Gaussian Mixture Reduction
}

\author{
Andrew R. Runnalls
}

\begin{abstract}
(C) 2006 IEEE. Personal use of this material is permitted. However, permission to reprint/republish this material for advertising or promotional purposes or for creating new collective works for resale or redistribution to servers or lists, or to reuse any copyrighted component of this work in other works must be obtained from the IEEE.

This material is presented to ensure timely dissemination of scholarly and technical work. Copyright and all rights therein are retained by authors or by other copyright holders. All persons copying this information are expected to adhere to the terms and constraints invoked by each author's copyright. In most cases, these works may not be reposted without the explicit permission of the copyright holder.
\end{abstract}

Abstract-A common problem in multi-target tracking is to approximate a Gaussian mixture by one containing fewer components; similar problems can arise in integrated navigation. A common approach is successively to merge pairs of components, replacing the pair with a single Gaussian component whose moments up to second order match those of the merged pair. Salmond [1] and Williams [2], [3] have each proposed algorithms along these lines, but using different criteria for selecting the pair to be merged at each stage. The paper shows how under certain circumstances each of these pair-selection criteria can give rise to anomalous behaviour, and proposes that a key consideration should be the Kullback-Leibler discrimination of the reduced mixture with respect to the original mixture. Although computing this directly would normally be impractical, the paper shows how an easily-computed upper bound can be used as a pair-selection criterion which avoids the anomalies of the earlier approaches. The behaviour of the three algorithms is compared using a highdimensional example drawn from terrain-referenced navigation.

Index Terms-Gaussian mixture, data fusion, integrated navigation, tracking.

\section{INTRODUCTION}

$\mathbf{S}$ EVERAL data fusion algorithms, usually derived in some way from the Kalman filter, represent the state of the observed system as a mixture of Gaussian distributions. An important example is the multiple hypothesis approach to tracking multiple targets where there is ambiguity in assigning observations to tracks—-see for example [4, Sec. 6.7]—and this is the application motivating Salmond's and Williams's papers cited below. However, Gaussian mixture approaches are also useful in integrated navigation applications where, for example, there is some ambiguity in the position fixes used to augment an inertial navigation system: this is the application motivating the present note [5], [6].

Andrew Runnalls is with the Computing Laboratory at the University of Kent, Canterbury CT2 7NF, Kent, UK. (e-mail: A.R.Runnalls@kent.ac.uk).
A common drawback with these Gaussian mixture algorithms is that there is a tendency for the number of components of the mixture to grow without bound: indeed, if the algorithm were to simply to follow the statistical model on which the method is based, the number of components would increase exponentially over time. To combat this, various pragmatic measures must be taken to keep the number of components in check. Typically this will be achieved either by discarding components with low probability, and/or by merging components which represent similar state hypotheses.

Salmond [1] proposed a mixture reduction algorithm in which the number of components is reduced by repeatedly choosing the two components that appear to be most similar to each other, and merging them. His criterion of similarity is based on concepts from the statistical analysis of variance, and seeks to minimise the increase in 'within-component' variance resulting from merging the two chosen components.

Williams [2], [3] proposed a mixture reduction algorithm based on an integrated squared difference (ISD) similarity measure, which as he points out has the big advantage that the similarity between two arbitrary Gaussian mixtures can be expressed in closed form. The algorithm he proposes uses a hill-climbing optimisation to search for a reduced mixture with the greatest similarity to the original mixture; however, to find starting points for the optimisation process, he uses a pairwise merge algorithm similar to Salmond's, but using the ISD similarity measure.

In the present paper, we propose a third variation on the pairwise-merge approach, in which the measure of similarity between two components is based on the Kullback-Leibler (KL) discrimination measure [7].

The layout is as follows: Sec. II introduces a brief notation for Gaussian mixtures, defines the concept of a momentpreserving merge of two or more components of such a mixture, and outlines the pairwise-merge type of mixture reduction algorithm being considered in this paper. Sec. III introduces the KL discrimination measure. Sec. IV describes the criterion proposed in [1] for selecting which pair of components to merge at each stage, and identifies two properties of this criterion that may be considered anomalous. Sec. V similarly studies the ISD criterion proposed by Williams, and identifies a property of this criterion that may be considered undesirable in some applications, particularly where the system state vector has high dimensionality. Sec. VI proposes a dissimilarity measure for pair selection based on KL discrimination, and explores its properties; Sec. VII then discusses the advantages and disadvantages of a pairwise merge algorithm based on this dissimilarity measure. Sec. VIII compares the operation of the Salmond, Williams, and KL reduction algorithms in reducing 
a high-dimensional mixture arising in terrain-referenced navigation. Finally Sec. IX draws conclusions.

\section{General BACKGROUND}

\section{A. Notation}

We shall represent a component of a Gaussian mixture using notation of the form $(w, \boldsymbol{\mu}, P)$ : this represents a component with non-negative weight $w$, mean vector $\boldsymbol{\mu}$ and covariance matrix $P$. (We shall assume throughout that components' covariance matrices are strictly positive definite, and not merely non-negative definite.) We shall use notation such as $\left\{\left(w_{1}, \boldsymbol{\mu}_{1}, P_{1}\right),\left(w_{2}, \boldsymbol{\mu}_{2}, P_{2}\right), \ldots\left(w_{n}, \boldsymbol{\mu}_{n}, P_{n}\right)\right\}$ to denote a mixture of $n$ such components; such a mixture must satisfy $w_{1}+\cdots+w_{n}=1$, and has probability density function:

$f(\mathbf{x})=\sum_{i=1}^{n} \frac{w_{i}}{\sqrt{(2 \pi)^{d} \operatorname{det} P_{i}}} \exp \left[-\frac{1}{2}\left(\mathbf{x}-\boldsymbol{\mu}_{i}\right)^{T} P_{i}^{-1}\left(\mathbf{x}-\boldsymbol{\mu}_{i}\right)\right]$

where $d$ is the dimensionality of the state vector $\mathbf{x}$. A plain (unmixed) Gaussian distribution will be written using notation such as $\{(1, \mu, P)\}$.

\section{B. Merging Two Components}

Suppose we are given a mixture of two Gaussian components:

$$
\left\{\left(w_{1}, \boldsymbol{\mu}_{1}, P_{1}\right),\left(w_{2}, \boldsymbol{\mu}_{2}, P_{2}\right)\right\}
$$

(where $w_{1}+w_{2}=1$ ) and that we wish to approximate this mixture as a single Gaussian. A strong candidate is the Gaussian whose zeroth, first and second-order moments match those of (1), i.e. the Gaussian with mean vector $\boldsymbol{\mu}$ and covariance matrix $P$ as follows:

$$
\begin{aligned}
\boldsymbol{\mu}= & w_{1} \boldsymbol{\mu}_{1}+w_{2} \boldsymbol{\mu}_{2} \\
P= & w_{1}\left(P_{1}+\left(\boldsymbol{\mu}_{1}-\boldsymbol{\mu}\right)\left(\boldsymbol{\mu}_{1}-\boldsymbol{\mu}\right)^{T}\right) \\
& +w_{2}\left(P_{2}+\left(\boldsymbol{\mu}_{2}-\boldsymbol{\mu}\right)\left(\boldsymbol{\mu}_{2}-\boldsymbol{\mu}\right)^{T}\right) \\
= & w_{1} P_{1}+w_{2} P_{2}+w_{1} w_{2}\left(\boldsymbol{\mu}_{1}-\boldsymbol{\mu}_{2}\right)\left(\boldsymbol{\mu}_{1}-\boldsymbol{\mu}_{2}\right)^{T}
\end{aligned}
$$

(Theorem 3.2 will show that $\{(1, \boldsymbol{\mu}, P)\}$ is the Gaussian whose Kullback-Leibler discrimination from the mixture (1) is minimal.)

We shall refer to $(1, \boldsymbol{\mu}, P)$ as the moment-preserving merge of $\left(w_{1}, \boldsymbol{\mu}_{1}, P_{1}\right)$ and $\left(w_{2}, \boldsymbol{\mu}_{2}, P_{2}\right)$. More generally, we can remove the restriction that $w_{1}+w_{2}=1$ : given two weighted Gaussian components $\left(w_{i}, \boldsymbol{\mu}_{i}, P_{i}\right)$ and $\left(w_{j}, \boldsymbol{\mu}_{j}, P_{j}\right)$, with $w_{1}+w_{2} \leq 1$, their moment-preserving merge is the Gaussian component $\left(w_{i j}, \boldsymbol{\mu}_{i j}, P_{i j}\right)$ as follows (cf. [3, Eqs 24]):

$$
\begin{aligned}
w_{i j}= & w_{i}+w_{j} \\
\boldsymbol{\mu}_{i j}= & w_{i \mid i j} \boldsymbol{\mu}_{i}+w_{j \mid i j} \boldsymbol{\mu}_{j} \\
P_{i j}= & w_{i \mid i j} P_{i}+w_{j \mid i j} P_{j} \\
& +w_{i \mid i j} w_{j \mid i j}\left(\boldsymbol{\mu}_{i}-\boldsymbol{\mu}_{j}\right)\left(\boldsymbol{\mu}_{i}-\boldsymbol{\mu}_{j}\right)^{T}
\end{aligned}
$$

where we write $w_{i \mid i j}=w_{i} /\left(w_{i}+w_{j}\right)$ and $w_{j \mid i j}=w_{j} /\left(w_{i}+\right.$ $\left.w_{j}\right)$.

\section{Mixture Reduction Algorithm}

Suppose that we are given a mixture with $n$ components, and we wish to approximate it by a mixture of $m$ components, where $m \leq n$. In this paper, we focus on algorithms which operate in the following general way:

While more than $m$ components remain, choose the two components that in a sense to be defined are least dissimilar, and replace them by their momentpreserving merge.

The algorithm proposed in [1, Sec. 4] is of this type, using the dissimilarity measure to be described in Sec. IV; the algorithm proposed in [2], [3] uses an algorithm of this type to determine starting points for an optimisation procedure.

\section{KULlBACK-LEIBLER DiscRIMINATION}

If $f_{1}(\mathbf{x})$ and $f_{2}(\mathbf{x})$ are probability density functions over $\Re^{d}$, the Kullback-Leibler (KL) discrimination ${ }^{1}$ of $f_{2}$ from $f_{1}$ is defined as:

$$
d_{\mathrm{kl}}\left(f_{1}, f_{2}\right)=\int_{\Re^{d}} f_{1}(\mathbf{x}) \log \frac{f_{1}(\mathbf{x})}{f_{2}(\mathbf{x})} \mathrm{d} \mathbf{x}
$$

Although clearly $d_{\mathrm{kl}}(f, f)=0$, and $d_{\mathrm{kl}}(f, g) \geq 0$ (cf. [8, Theorem 2.6.3], [9, Theorem 4.3.1]), in general it is not true that $d_{\mathrm{kl}}(f, g)=d_{\mathrm{kl}}(g, f)$, nor that $d_{\mathrm{kl}}(f, g)+d_{\mathrm{kl}}(g, h) \geq$ $d_{\mathrm{kl}}(f, h)$.

To give an informal motivation for KL discrimination, suppose that we have a stream of data $x_{1}, x_{2}, \ldots$ which we assume to be independent samples either from $f(x)$ or from $g(x)$, and we wish to decide which. From a Bayesian perspective, the approach we might take is to continue drawing samples until the likelihood ratio $\prod_{i}\left(f\left(x_{i}\right) / g\left(x_{i}\right)\right)$ exceeds some predefined threshold, say 100:1 in favour on one candidate or the other. Equivalently, we will be aiming to achieve a sample large enough that the logarithm of the likelihood ratio falls outside the bounds $\pm \log 100$. Now suppose that (unknown to us) the data stream is actually coming from $f(x)$. Then the expected value of the log-likelihood-ratio for a single sample point will be $E(\log (f(x) / g(x)))=d_{\mathrm{kl}}(f, g)$. Consequently, the expected log-likelihood-ratio for the full sample will exceed $\log 100$ provided the sample size exceeds $(\log 100) / d_{\mathrm{kl}}(f, g)$. Roughly speaking, small values of $d_{\mathrm{kl}}(f, g)$ mean that we will need large samples to distinguish $f$ from $g$, and conversely.

The remainder of this section introduces theorems about Kullback-Leibler discrimination that we shall use in Sec. VI, and can be skipped on a first reading.

Theorem 3.1: Let $g_{1}(\mathbf{x})$ be the d-dimensional Gaussian pdf with mean vector $\boldsymbol{\mu}_{1}$ and positive definite covariance matrix $P_{1}$, and let $g_{2}(\mathbf{x})$ be the d-dimensional Gaussian pdf with mean vector $\boldsymbol{\mu}_{2}$ and p.d. covariance matrix $P_{2}$. Then:

$$
\begin{aligned}
& 2 d_{\mathrm{kl}}\left(g_{1}, g_{2}\right) \\
& =\operatorname{tr}\left(P_{2}^{-1}\left[P_{1}-P_{2}+\left(\boldsymbol{\mu}_{1}-\boldsymbol{\mu}_{2}\right)\left(\boldsymbol{\mu}_{1}-\boldsymbol{\mu}_{2}\right)^{T}\right]\right) \\
& \quad+\log \frac{\operatorname{det}\left(P_{2}\right)}{\operatorname{det}\left(P_{1}\right)}
\end{aligned}
$$

${ }^{1}$ Also referred to as cross-entropy, Kullback-Leibler information, or Kullback-Leibler divergence. However, Kullback and Leibler themselves [7] and several subsequent authors use the term 'divergence' to refer to $d_{\mathrm{kl}}\left(f_{1}, f_{2}\right)+d_{\mathrm{kl}}\left(f_{2}, f_{1}\right)$. It is also sometimes called the Kullback-Leibler distance, despite not satisfying the usual requirements for a distance measure. 
For a proof see for example [9, Theorem 7.2.8].

Theorem 3.2: Let $f(\mathbf{x})$ be a probability density function over $d$ dimensions with well-defined mean $\boldsymbol{\mu}_{*}$ and covariance matrix $P_{*}$, where $P_{*}$ is strictly positive-definite. As before, let $(1, \boldsymbol{\mu}, P)$ denote the Gaussian density with mean $\boldsymbol{\mu}$ and p.d. covariance matrix $P$. Then the unique minimum value of $d_{\mathrm{kl}}(f,(1, \boldsymbol{\mu}, P))$ is achieved when $\boldsymbol{\mu}=\boldsymbol{\mu}_{*}$ and $P=P_{*}$.

For a proof see the Appendix.

Theorem 3.3: If $f(\mathbf{x}), h_{1}(\mathbf{x})$ and $h_{2}(\mathbf{x})$ are any pdfs over $d$ dimensions and $0 \leq w \leq 1$ then, writing $\bar{w}$ for $1-w$ :

$$
\begin{aligned}
& d_{\mathrm{kl}}\left(w h_{1}+\bar{w} h_{2}, f\right) \leq w d_{\mathrm{kl}}\left(h_{1}, f\right)+\bar{w} d_{\mathrm{kl}}\left(h_{2}, f\right) \\
& d_{\mathrm{kl}}\left(f, w h_{1}+\bar{w} h_{2}\right) \leq w d_{\mathrm{kl}}\left(f, h_{1}\right)+\bar{w} d_{\mathrm{kl}}\left(f, h_{2}\right)
\end{aligned}
$$

This is a standard result: for a proof see [9, Theorem 4.3.2] or [8, Theorem 2.7.2].

Theorem 3.4: If $f_{1}(\mathbf{x}), f_{2}(\mathbf{x})$ and $h(\mathbf{x})$ are any $p d f$ s over $d$ dimensions, $0 \leq w \leq 1$ and $\bar{w}=1-w$, then:

$$
d_{\mathrm{kl}}\left(w f_{1}+\bar{w} h, w f_{2}+\bar{w} h\right) \leq w d_{\mathrm{kl}}\left(f_{1}, f_{2}\right)
$$

For a proof see the Appendix.

\section{SALMOND's CRITERION}

Let $\left\{\left(w_{1}, \boldsymbol{\mu}_{1}, P_{1}\right), \ldots\left(w_{n}, \boldsymbol{\mu}_{n}, P_{n}\right)\right\}$ be an $n$-component Gaussian mixture, and let $\boldsymbol{\mu}$ and $P$ be respectively the overall mean and the overall variance of this mixture. Clearly

$$
\boldsymbol{\mu}=\sum_{i=1}^{n} w_{i} \boldsymbol{\mu}_{i}
$$

while $P$ can be written as $P=W+B$ where $W$ is the 'within-components' contribution to the total variance, given by:

$$
W=\sum_{i=1}^{n} w_{i} P_{i}
$$

while $B$ is the 'between-components' contribution given by:

$$
B=\sum_{i=1}^{n} w_{i}\left(\boldsymbol{\mu}_{i}-\boldsymbol{\mu}\right)\left(\boldsymbol{\mu}_{i}-\boldsymbol{\mu}\right)^{T}
$$

When two components are replaced by their momentpreserving merge, the effect is, roughly speaking, to increase $W$ and decrease $B$ by a corresponding amount, leaving the total variance $P$ unchanged. Salmond's general idea [1, Sec. 4] is to choose for merging two components $i$ and $j$ such that the increase in $W$ is minimised. He shows that the change in $W$ when components $i$ and $j$ are replaced by their momentpreserving merge is

$$
\Delta W_{i j}=\frac{w_{i} w_{j}}{w_{i}+w_{j}}\left(\boldsymbol{\mu}_{i}-\boldsymbol{\mu}_{j}\right)\left(\boldsymbol{\mu}_{i}-\boldsymbol{\mu}_{j}\right)^{T}
$$

However, $\Delta W_{i j}$ is a matrix, whereas we require a scalar dissimilarity measure. Salmond proposes using the following measure:

$$
D_{\mathrm{S}}^{2}(i, j)=\operatorname{tr}\left(P^{-1} \Delta W_{i j}\right)
$$

Here the trace reduces its matrix argument to a scalar, and the premultiplication by $P^{-1}$ ensures that the resulting dissimilarity measure is invariant under linear transformations of the state space.

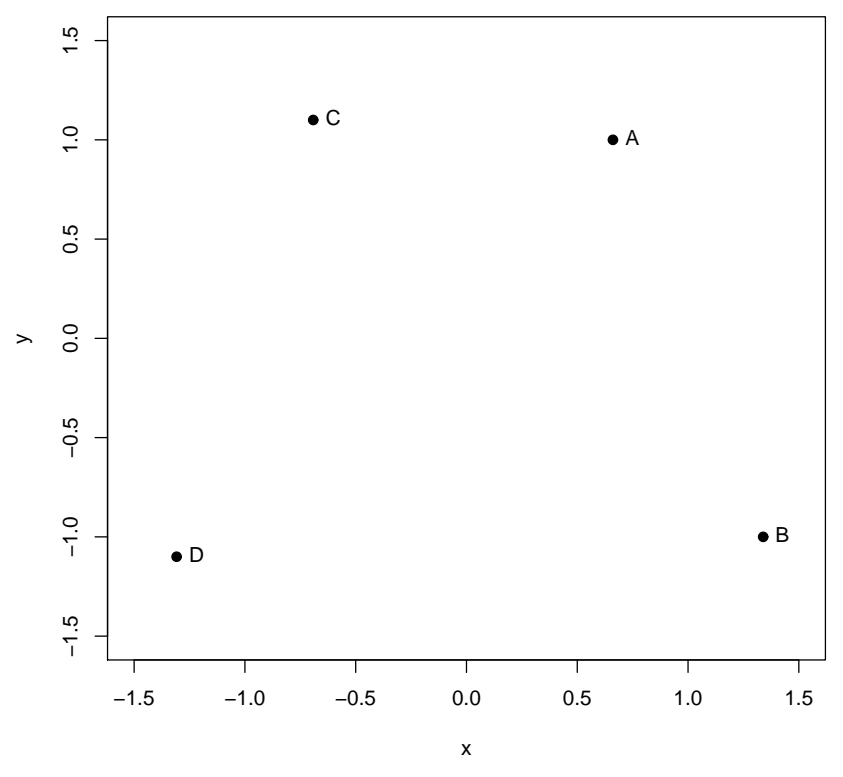

Fig. 1. Means of the components in Example 4.2

However, the dissimilarity measure defined in (6) has two properties that may be considered undesirable as a basis for choosing which components to merge. First, the measure depends on the means of the components, but not on their individual covariance matrices, leading to the behaviour in this example:

Example 4.1: A mixture comprises three two-dimensional components $\left\{\left(\frac{1}{3}, \boldsymbol{\mu}, P_{1}\right),\left(\frac{1}{3}, \boldsymbol{\mu}+\delta \boldsymbol{\mu}, P_{1}\right),\left(\frac{1}{3}, \boldsymbol{\mu}, P_{2}\right)\right\}$, where $\delta \boldsymbol{\mu}$ is very small $\left(\right.$ e.g. $\left.\delta \boldsymbol{\mu}=(0.0001,0.0001)^{T}\right)$ but $P_{2}$ is very different from $P_{1}$ :

$$
P_{1}=\left(\begin{array}{cc}
1 & 0.9 \\
0.9 & 1
\end{array}\right) \quad P_{2}=\left(\begin{array}{cc}
1 & -0.9 \\
-0.9 & 1
\end{array}\right)
$$

We wish to reduce the mixture to two components. Then, using (6), we will choose to merge the first and third components, yielding a merged component $\left(\frac{2}{3}, \boldsymbol{\mu}, I_{2}\right)$, where $I_{2}$ is the twodimensional identity matrix.

The reader may well consider that in this example it would be better to merge the first two components, yielding $\left(\frac{2}{3}, \boldsymbol{\mu}+\right.$ $\left.\frac{1}{2} \delta \boldsymbol{\mu}, P_{1}+\frac{1}{4} \delta \boldsymbol{\mu} \delta \boldsymbol{\mu}^{T}\right)$.

The second drawback arises from the presence of the overall covariance $P$ within (6). This has the implication that adding a new component to a mixture may alter the order in which the existing components are merged, as shown in the following example.

Example 4.2: A mixture over the two dimensions $(x, y)$ consists of four components

$$
\begin{aligned}
& A=\left(0.25,(0.661,1)^{T}, I_{2}\right) \\
& B=\left(0.25,(1.339,-1)^{T}, I_{2}\right) \\
& C=\left(0.25,(-0.692,1.1)^{T}, I_{2}\right) \\
& D=\left(0.25,(-1.308,-1.1)^{T}, I_{2}\right)
\end{aligned}
$$


(The means of the components are shown in Fig. 1.) We wish to reduce this mixture to three components. It is readily established that the overall mean of the mixture is $(0,0)^{T}$, and its covariance matrix is $2.105 I_{2}$. From the latter fact, it follows that criterion (6) will lead us simply to merge the two components whose means are closest together, namely $A$ and C.

Now modify the original mixture by reducing the weights of components $A$ to $D$ to 0.2 , and adding a fifth component $E=\left(0.2,(0,-10)^{T}, I_{2}\right)$. We wish to reduce this new mixture to three components. It turns out that criterion (6) now selects components $A$ and $B$ for the first merge, and components $C$ and $D$ for the second merge. This is because, although $E$ is a weak candidate for either merge, its inclusion in the mixture has greatly increased its overall variance in the $y$-direction, meaning that (6) now weights differences in $x$ more heavily than differences in $y$.

\section{WILliams's CRITERION}

Williams [2] and Williams and Maybeck [3] propose a method of Gaussian mixture reduction based on the integrated squared difference (ISD) measure of the dissimilarity between two pdfs $f_{1}(\mathbf{x})$ and $f_{2}(\mathbf{x})$ :

$$
J_{S}=\int\left(f_{1}(\mathbf{x})-f_{2}(\mathbf{x})\right)^{2} d \mathbf{x}
$$

(cf. [3, Eq. 4]. This has the important property that the dissimilarity between two arbitrary Gaussian mixtures can be expressed in closed form (given in [3, Eq. 10]) — a property regrettably not shared by the measure proposed in the present paper.

Their algorithm for reducing an $n$-component mixture to an $m$-component mixture $(m \leq n)$ can be summarised as follows:

1) While more than $m$ components remain consider all possible operations of the following two kinds:

- Deleting a component and renormalising the remaining mixture;

- Replacing a pair of components with their momentpreserving merge;

and in each case evaluate the ISD-dissimilarity of the resulting mixture from the original mixture. Apply the operation for which this dissimilarity is a minimum.

2) Use the resulting $m$-component mixture as the starting point for gradient-based optimisation technique, to seek an $m$-component mixture with lower dissimilarity to the original mixture.

The authors note that the optimisation at Step 2 will seek a local minimum rather than the global minimum: hence the need to choose the starting point carefully.

The ISD cost measure circumvents both of the drawbacks of Salmond's criterion. First, the measure depends explicitly on the covariance matrices as well as the means of the components. Second, the cost incurred by merging two components depends only on the parameters of those components, and not on other characteristics of the mixture of which they form a part. Consequently, the anomalies observed in Examples 4.1 and 4.2 do not arise.
However, the ISD criterion leads to puzzling behaviour of its own. To illustrate this, we will focus on mixtures where the components are radially symmetric, i.e. the covariance matrices are multiples of the identity matrix. Consider first the case where the starting mixture is $\left\{\left(w, \boldsymbol{\mu}-c \sigma \mathbf{u}, \sigma^{2} I_{d}\right),(w, \boldsymbol{\mu}+\right.$ $\left.\left.c \sigma \mathbf{u}, \sigma^{2} I_{d}\right)\right\}$, where $\boldsymbol{\mu}$ is arbitrary and $\mathbf{u}$ is a $d$-dimensional unit vector. The means of the two components of this mixture are distance $2 c \sigma$ apart.

In this case it follows from [3, Eq. 12] that the ISD cost of deleting one of the components (and raising the other component to unit weight) is given by:

$$
J_{S}=\frac{4 w^{2}}{\sigma^{d} \sqrt{(4 \pi)^{d}}} h_{D}(c)
$$

where

$$
h_{D}(c)=\frac{1}{2}\left(1-\exp \left(-c^{2}\right)\right)
$$

while the cost of replacing the two components by their moment-preserving merge, namely $\left(2 w, \boldsymbol{\mu}, \sigma^{2}\left(I+c^{2} \mathbf{u u}^{T}\right)\right)$, is:

$$
J_{S}=\frac{4 w^{2}}{\sigma^{d} \sqrt{(4 \pi)^{d}}} h_{M}(c)
$$

where

$$
\begin{aligned}
h_{M}(c)= & \frac{1}{2}\left(1+\exp \left(-c^{2}\right)\right)+\frac{1}{\sqrt{1+c^{2}}} \\
& -\frac{2 \sqrt{2}}{\sqrt{2+c^{2}}} \exp \left(-\frac{c^{2}}{2\left(2+c^{2}\right)}\right)
\end{aligned}
$$

The functions $h_{M}(c)$ and $h_{D}(c)$ are both zero for $c=0$ and as $c$ increases, both functions increase monotonically, tending towards $\frac{1}{2}$ as $c \rightarrow \infty$. It can be shown that $h_{D}(c)>h_{M}(c)$ except when $c$ is zero, so the deletion option will not be considered further.

In the example under consideration, $\sigma$ acts simply as a scale factor, but it nevertheless appears in (13), raised moreover to the power $d$. This leads to some surprising behaviour in the way in which Williams's algorithm selects pairwise merges, as in the following twelve-dimensional example. (It is not unusual in inertial navigation applications for the state vector to have 15 or more dimensions.)

Example 5.1: A mixture over the space $\left(x_{1}, \ldots x_{12}\right)$ comprises four components

$$
\begin{aligned}
& A=\left(0.25,(-20,-0.5,0, \ldots, 0)^{T}, I_{12}\right) \\
& B=\left(0.25,(-20,0.5,0, \ldots 0)^{T}, I_{12}\right) \\
& C=\left(0.25,(20,-10,0, \ldots 0)^{T}, 4 I_{12}\right) \\
& D=\left(0.25,(20,10,0, \ldots 0)^{T}, 4 I_{12}\right)
\end{aligned}
$$

where in each mean vector the ellipsis ... comprises eight zeroes. Note that components $A$ and $B$ have negligible probability within the region where $x_{1}>0$, and $C$ and $D$ have negligible probability within the region $x_{1}<0$.

Assume that we wish to reduce this four-component mixture to three components. Now, according to (13) the cost of replacing components $A$ and $B$ by their moment-preserving merge is

$$
J_{S}=\frac{1}{4(4 \pi)^{6}} h_{M}(0.5) \approx 6.39 \times 10^{-12}
$$


while the cost of replacing $C$ and $D$ by their momentpreserving merge is:

$$
J_{S}=\frac{1}{4 \times 2^{12}(4 \pi)^{6}} h_{M}(5) \approx 5.48 \times 10^{-12}
$$

Consequently, the Williams algorithm will choose to merge $C$ and $D$ rather than merging $A$ and $B$.

This is despite the fact that mixture of $A$ and $B$ is already unimodal, and is very similar in shape to their momentpreserving merge. In contrast, the mixture of $C$ and $D$ is decidedly bimodal, the means of these components being ten standard deviations apart.

In fact, direct numerical integration reveals that the Kullback-Leibler discrimination of the mixture $\{A, B, C D\}$ (where $C D$ is the result of merging $C$ and $D$ ) from the original mixture $\{A, B, C, D\}$ is 0.468 , so (following the discussion at the start of Sec. III) it would need only about 10 samples from the original mixture to distinguish it from $\{A, B, C D\}$ with a likelihood ratio of 100:1. In contrast, the discrimination of the mixture $\{A B, C, D\}$ from the original is only $7.52 \times 10^{-5}$, so requiring an average of over 60000 samples to achieve the same likelihood ratio.

The phenomenon illustrated by this example arises from the scale-dependency of the ISD cost measure, as exhibited by the presence of the scale factor $\sigma$ in the cost measure of (13). It is particularly pronounced in spaces of high dimensionality, and means that - at least in some applications - the ISD cost measure may not be suitable as a basis for Gaussian mixture reduction.

In Sec. VI we present an alternative criterion for mixture reduction which does not exhibit scale-dependency, and which also avoids the drawbacks of Salmond's criterion.

\section{A Dissimilarity MEASURE BASED ON KL DISCRIMINATION}

\section{A. Motivation}

At each iteration of the algorithm outlined in Sec. II-C, we wish to choose two components from the mixture for merging. Our ultimate objective is to find a weighted mixture of $m$ Gaussian components in such a way as to keep the KL discrimination of the $m$-component mixture from the original $n$-component mixture as small as possible, subject to being able to accomplish this with an algorithm that is computationally reasonably fast. A reasonable criterion, therefore, is to choose two components in such a way as to minimise the KL discrimination of the mixture after the merge from the mixture before the merge.

Unfortunately, there appears to be no closed-form expression for the KL discrimination of one (non-trivial) Gaussian mixture from another. (This fact deterred Williams [2, Sec. 3.3.1.4] from pursuing a cost measure based on KL discrimination; were it not for this, he says it would be the "ideal cost function" for Gaussian mixture reduction.) However, Sec. III provided two theorems that enable us to put an upper bound on the discrimination of the mixture after the merge from the mixture before the merge. This leads us to the dissimilarity measure $B\left(\left(w_{i}, \boldsymbol{\mu}_{i}, P_{i}\right),\left(w_{j}, \boldsymbol{\mu}_{j}, P_{j}\right)\right)$ now to be defined.

\section{B. Definition of $B\left(\left(w_{i}, \boldsymbol{\mu}_{i}, P_{i}\right),\left(w_{j}, \boldsymbol{\mu}_{j}, P_{j}\right)\right)$}

Theorem 3.4 tells us that the discrimination of the mixture after merging components $i$ and $j$ from the mixture before the merge will not exceed $w_{i}+w_{j}$ times the discrimination of the single Gaussian $\left\{\left(1, \boldsymbol{\mu}_{i j}, P_{i j}\right)\right\}$ from the (normalised) mixture $\left\{\left(w_{i \mid i j}, \boldsymbol{\mu}_{i}, P_{i}\right),\left(w_{j \mid i j}, \boldsymbol{\mu}_{j}, P_{j}\right)\right\}$. (Refer to Sec. II-B for notation.)

Moreover Theorem 3.3 tells us that this discrimination, which we shall write as

$$
d_{\mathrm{kl}}\left(\left\{\left(w_{i \mid i j}, \boldsymbol{\mu}_{i}, P_{i}\right),\left(w_{j \mid i j}, \boldsymbol{\mu}_{j}, P_{j}\right)\right\},\left\{\left(1, \boldsymbol{\mu}_{i j}, P_{i j}\right)\right\}\right)
$$

will not exceed

$$
\begin{aligned}
& \frac{1}{w_{i}+w_{j}}\left(w_{i} d_{\mathrm{kl}}\left(\left\{\left(1, \boldsymbol{\mu}_{i}, P_{i}\right)\right\},\left\{\left(1, \boldsymbol{\mu}_{i j}, P_{i j}\right)\right\}\right)\right. \\
& \left.+w_{j} d_{\mathbf{k l}}\left(\left\{\left(1, \boldsymbol{\mu}_{j}, P_{j}\right)\right\},\left\{\left(1, \boldsymbol{\mu}_{i j}, P_{i j}\right)\right\}\right)\right)
\end{aligned}
$$

Putting these together, it follows that the discrimination of the mixture following the merge from the mixture before the merge will not exceed:

$$
\begin{aligned}
& B\left(\left(w_{i}, \boldsymbol{\mu}_{i}, P_{i}\right),\left(w_{j}, \boldsymbol{\mu}_{j}, P_{j}\right)\right) \\
& =w_{i} d_{\mathrm{kl}}\left(\left\{\left(1, \boldsymbol{\mu}_{i}, P_{i}\right)\right\},\left\{\left(1, \boldsymbol{\mu}_{i j}, P_{i j}\right)\right\}\right) \\
& \quad+w_{j} d_{\mathrm{kl}}\left(\left\{\left(1, \boldsymbol{\mu}_{j}, P_{j}\right)\right\},\left\{\left(1, \boldsymbol{\mu}_{i j}, P_{i j}\right)\right\}\right)
\end{aligned}
$$

We now show how this upper bound $B\left(\left(w_{i}, \boldsymbol{\mu}_{i}, P_{i}\right),\left(w_{j}, \boldsymbol{\mu}_{j}, P_{j}\right)\right)$ can be computed in practice. From Theorem 3.1, we have:

$$
\begin{aligned}
& 2 d_{\mathrm{kl}}\left(\left\{\left(1, \boldsymbol{\mu}_{i}, P_{i}\right)\right\},\left\{\left(1, \boldsymbol{\mu}_{i j}, P_{i j}\right)\right\}\right) \\
& =\operatorname{tr}\left(P_{i j}^{-1}\left[P_{i}-P_{i j}+\left(\boldsymbol{\mu}_{i}-\boldsymbol{\mu}_{i j}\right)\left(\boldsymbol{\mu}_{i}-\boldsymbol{\mu}_{i j}\right)^{T}\right]\right) \\
& \quad+\log \frac{\operatorname{det}\left(P_{i j}\right)}{\operatorname{det}\left(P_{i}\right)} \\
& =\operatorname{tr}\left(P_{i j}^{-1}\left[P_{i}-P_{i j}+w_{j \mid i j}^{2}\left(\boldsymbol{\mu}_{i}-\boldsymbol{\mu}_{j}\right)\left(\boldsymbol{\mu}_{i}-\boldsymbol{\mu}_{j}\right)^{T}\right]\right) \\
& \quad+\log \operatorname{det}\left(P_{i j}\right)-\log \operatorname{det}\left(P_{i}\right)
\end{aligned}
$$

A corresponding expression can be obtained for $2 d_{\mathrm{kl}}\left(\left\{\left(1, \boldsymbol{\mu}_{j}, P_{j}\right)\right\},\left\{\left(1, \boldsymbol{\mu}_{i j}, P_{i j}\right)\right\}\right)$ by replacing $P_{i}$ by $P_{j}$ and $w_{j \mid i j}$ by $w_{i \mid i j}$.

Consequently, substituting into (19) and using the fact that trace is a linear operator, we find:

$$
\begin{aligned}
2 B\left(\left(w_{i}, \boldsymbol{\mu}_{i}, P_{i}\right),\left(w_{j}, \boldsymbol{\mu}_{j}, P_{j}\right)\right) \\
=\operatorname{tr}\left(P_{i j}^{-1} \breve{P}_{i j}\right)+\left(w_{i}+w_{j}\right) \log \operatorname{det}\left(P_{i j}\right) \\
\quad-w_{i} \log \operatorname{det}\left(P_{i}\right)-w_{j} \log \operatorname{det}\left(P_{j}\right)
\end{aligned}
$$

where

$$
\begin{aligned}
\breve{P}_{i j}= & w_{i} P_{i}+w_{j} P_{j}-\left(w_{i}+w_{j}\right) P_{i j} \\
& +\frac{w_{i} w_{j}}{w_{i}+w_{j}}\left(\boldsymbol{\mu}_{i}-\boldsymbol{\mu}_{j}\right)\left(\boldsymbol{\mu}_{i}-\boldsymbol{\mu}_{j}\right)^{T}
\end{aligned}
$$

If we now substitute the expression for $P_{i j}$ in (4) into (20), we find that $\breve{P}_{i j}$ equals zero. Therefore:

$$
\begin{aligned}
& B\left(\left(w_{i}, \boldsymbol{\mu}_{i}, P_{i}\right),\left(w_{j}, \boldsymbol{\mu}_{j}, P_{j}\right)\right) \\
& =\frac{1}{2}\left[\left(w_{i}+w_{j}\right) \log \operatorname{det}\left(P_{i j}\right)\right. \\
& \left.\quad \quad-w_{i} \log \operatorname{det}\left(P_{i}\right)-w_{j} \log \operatorname{det}\left(P_{j}\right)\right]
\end{aligned}
$$




\section{Properties of $B(i, j)$}

In the remainder of this paper we shall write $B(i, j)$ as a shorthand for $B\left(\left(w_{i}, \boldsymbol{\mu}_{i}, P_{i}\right),\left(w_{j}, \boldsymbol{\mu}_{j}, P_{j}\right)\right)$.

First of all, it is clear that the function is symmetric: $B(i, j)=B(j, i)$.

In one dimension, with $P_{i}=\left(\sigma_{i}^{2}\right), P_{j}=\left(\sigma_{j}^{2}\right)$, becomes:

$$
\begin{gathered}
\frac{2 B(i, j)}{w_{i}+w_{j}}=\log \left[w_{i \mid i j}\left(\frac{\sigma_{i}^{2}}{\sigma_{j}^{2}}\right)^{w_{j \mid i j}}+w_{j \mid i j}\left(\frac{\sigma_{j}^{2}}{\sigma_{i}^{2}}\right)^{w_{i \mid i j}}\right. \\
\left.+w_{i \mid i j} w_{j \mid i j} \frac{\left(\mu_{i}-\mu_{j}\right)^{2}}{\sigma_{i}^{2 w_{i \mid i j}} \sigma_{j}^{2 w_{j \mid i j}}}\right]
\end{gathered}
$$

showing that in this case $B(i, j)$ depends only on the weights $w_{i}, w_{j}$ and on the dimensionless quantities $\sigma_{i}^{2} / \sigma_{j}^{2}$ and $\left(\mu_{i}-\right.$ $\left.\mu_{j}\right) /\left(\sigma_{i}^{w_{i \mid i j}} \sigma_{j}^{w_{j \mid i j}}\right)$.

This conclusion can be extended to more than one dimension using a simultaneous diagonalisation procedure. Since $P_{i}$ and $P_{j}$ are both positive definite, by a small variation of the procedure described in [10, Sec. 1c.3(ii)] we can find a square unitary $^{2}$ matrix $U_{i j}$ and diagonal matrices $D_{i}$ and $D_{j}$, with all their diagonal elements positive, such that:

$$
P_{i}=U_{i j}^{-1} D_{i} U_{i j}^{-T} \quad P_{j}=U_{i j}^{-1} D_{j} U_{i j}^{-T}
$$

Substituting from these equations into (4) we get:

$$
\begin{gathered}
P_{i j}=w_{i \mid i j} U_{i j}^{-1} D_{i} U_{i j}^{-T}+w_{j \mid i j} U_{i j}^{-1} D_{j} U_{i j}^{-T} \\
+w_{i \mid i j} w_{j \mid i j}\left(\boldsymbol{\mu}_{i}-\boldsymbol{\mu}_{j}\right)\left(\boldsymbol{\mu}_{i}-\boldsymbol{\mu}_{j}\right)^{T} \\
=U_{i j}^{-1}\left(w_{i \mid i j} D_{i}+w_{j \mid i j} D_{j}\right. \\
\left.\quad+w_{i \mid i j} w_{j \mid i j} \mathbf{u}_{i j} \mathbf{u}_{i j}^{T}\right) U_{i j}^{-T}
\end{gathered}
$$

where we have written $\mathbf{u}_{i j}$ for $U_{i j}\left(\boldsymbol{\mu}_{i}-\boldsymbol{\mu}_{j}\right)$. Now, substituting from (23) and (24) into (21) and using the fact that $\operatorname{det}(A B)=$ $\operatorname{det} A \times \operatorname{det} B$, we get:

$$
\begin{gathered}
\frac{2 B(i, j)}{w_{i}+w_{j}} \\
=\quad \log \operatorname{det}\left(w_{i \mid i j} D_{i}+w_{j \mid i j} D_{j}+w_{i \mid i j} w_{j \mid i j} \mathbf{u}_{i j} \mathbf{u}_{i j}^{T}\right) \\
\quad-w_{i \mid i j} \log \operatorname{det}\left(D_{i}\right)-w_{j \mid i j} \log \operatorname{det}\left(D_{j}\right) \\
=\quad \log \operatorname{det}\left(w_{i \mid i j} D_{i}+w_{j \mid i j} D_{j}+w_{i \mid i j} w_{j \mid i j} \mathbf{u}_{i j} \mathbf{u}_{i j}^{T}\right) \\
\quad-\log \operatorname{det}\left(D_{i}^{w_{i \mid i j}}\right)-\log \operatorname{det}\left(D_{j}^{w_{j \mid i j}}\right) \\
=\quad \log \operatorname{det}\left(D_{i}^{-w_{i \mid i j}} D_{j}^{-w_{j \mid i j}}\right. \\
\left.\quad\left[w_{i \mid i j} D_{i}+w_{j \mid i j} D_{j}+w_{i \mid i j} w_{j \mid i j} \mathbf{u}_{i j} \mathbf{u}_{i j}^{T}\right]\right) \\
=\quad \log \operatorname{det}\left(w_{i \mid i j}\left(D_{i} D_{j}^{-1}\right)^{w_{j \mid i j}}+w_{j \mid i j}\left(D_{j} D_{i}^{-1}\right)^{w_{i \mid i j}}\right. \\
\left.\quad+w_{i \mid i j} w_{j \mid i j} D_{i}^{-w_{i \mid i j}} D_{j}^{-w_{j \mid i j}} \mathbf{u}_{i j} \mathbf{u}_{i j}^{T}\right)
\end{gathered}
$$

where the notation $D^{\alpha}$ denotes the diagonal matrix whose elements are the corresponding elements of $D$ raised to the power $\alpha$. Thus $B(i, j)$ depends only on the weights $w_{i}, w_{j}$ and the dimensionless quantities $D_{i} D_{j}^{-1}$ and $D_{i}^{-w_{i \mid i j} / 2} D_{j}^{-w_{j \mid i j} / 2} \mathbf{u}_{i j}$.

\footnotetext{
${ }^{2}$ i.e. a matrix with determinant unity. Note that the notation $U$ stands for 'unitary': $U_{i j}$ is not in general upper triangular.
}

By inspection of (25) it can be seen that $B(i, j)=0$ if and only if at least one of the following three conditions holds: (a) $w_{i}=0,(b) w_{j}=0$, or (c) $\boldsymbol{\mu}_{i}=\boldsymbol{\mu}_{j}$ and $P_{i}=P_{j}$. A counterexample to the triangle inequality is given (in one dimension) by putting $w_{1}=w_{2}=w_{3}=\frac{1}{3}, \mu_{1}=\mu_{2}=\mu_{3}$ and $\sigma_{3}=2 \sigma_{2}=4 \sigma_{1}$; then from (22) we have $B(1,2)=$ $B(2,3) \approx 0.07$ but $B(1,3) \approx 0.25$.

\section{DISCUSSION}

We propose that, in each iteration of the algorithm outlined in Sec. II-C, we select for merging two components $i$ and $j$, $i \neq j$, such that $B(i, j)$ is minimised. The dissimilarity measure $B(i, j)$ as given by (21) is reasonably easy to compute, with computational complexity at most $\mathcal{O}\left(d^{3}\right)$. Consequently, if our task is to reduce a mixture of $n$ components to a mixture with $c n$ components, where $c<1$ is a constant, this will have total computational complexity of $\mathcal{O}\left(n^{3} d^{3}\right)$.

This criterion has qualitatively the right properties. Roughly speaking, it will tend to select for merging:

1) Components with low weights. Note how the weights appear outside the logarithms in (21), and so can have a dominant effect.

2) Components whose means are close together in relation to their variances, as measured by the length of the vector $D_{i}^{-w_{i \mid i j} / 2} D_{j}^{-w_{j \mid i j} / 2} \mathbf{u}_{i j}$ (cf. (25)).

3) Components whose covariance matrices are similar, in the sense that the term $D_{i} D_{j}^{-1}$ in (25) is close to the identity matrix.

The $B(i, j)$ criterion avoids the drawbacks of Salmond's criterion, in that $(a)$ it depends explicitly on the covariance matrices of components $i$ and $j$, and will avoid merging components where these are very different, and $(b)$ adding a new component to a mixture cannot alter the order in which existing components are merged. Nor does the $B(i, j)$ criterion exhibit the scale dependency of the ISD measure; for example, corresponding to (13), we get simply

$$
B(i, j)=w \log \left(1+c^{2}\right)
$$

which does not depend on $\sigma$, or indeed on $d$. Consequently, in Example 5.1 we have $B(C, D) / B(A, B) \approx 14.6$, so $A$ would certainly be merged with $B$ in preference to merging $C$ and $D$.

We make no claims for optimality for the resulting algorithm, but it is straightforward, and at each iteration we know that the Kullback-Leibler discrimination of the post-iteration mixture from the pre-iteration mixture cannot exceed $B(i, j)$.

The fact that $B(i, j)$ is merely an upper bound on the $\mathrm{K}$ $\mathrm{L}$ discrimination, rather than an exact value, is admittedly a drawback. Moreover, since Kullback-Leibler discrimination does not satisfy the triangle inequality, there is no simple way of bounding the discrimination that arises over the course of two or more iterations of the algorithm. However, obtaining a direct estimate of the K-L bound would appear to require a numerical method, e.g. numerical integration. Worse, this integration would need to be carried out multiple times: $\mathcal{O}\left(n^{3}\right)$ times if, as above, our task is to reduce $n$ components to $\mathrm{cn}$ components. In many applications this will be computationally 
prohibitive. A possible compromise approach would be to use the $B(i, j)$ criterion to compile a shortlist of possible component merges, selection from within this shortlist being by direct numerical integration.

\section{A PRACTICAL EXAMPLE}

This section compares the operation of Salmond's criterion, the ISD measure and the merging criterion introduced in Sec. VI as applied to reducing a Gaussian mixture over 15 dimensions from its original 16 components down to four components. This dataset arises from an application to terrainreferenced navigation, specifically from the simulation run previously reported in [5, Fig. 7].

The state vector comprises three elements of position error (north, up, and down), three components of velocity error, three platform misalignment angles, three accelerometer biases and three gyro drift terms. However, for ease of visualisation, in this paper we shall illustrate the algorithms' operation by examining the marginal distribution over the two horizontal components of position error.

Panel (a) of Fig. 2 shows the starting mixture. Each Gaussian component is represented by an elliptical contour enclosing $50 \%$ of its probability volume. In each ellipse, a sector is shown shaded: the proportion of the area of the ellipse thus shaded represents the component's weight within the mixture. The thicker curves in the figure are two contours of the mixture as a whole: the dashed line encloses $95 \%$ of the mixture's volume, while the solid line encloses $50 \%$ of its volume (within two regions).

The mixture represents the navigation system's state estimate just a few seconds after terrain-referenced navigation started. Consequently there is still considerable uncertainty about the aircraft's position: the graticule in the figure comprises $1 \mathrm{~km}$ squares.

Panel (b), (c) and (d) show the result of reducing this mixture to four components using the algorithms considered earlier in the paper. In panel (b), the reduction uses Salmond's criterion. Panel (c) shows the result of applying the algorithm described in [3, Sec. 4], except that (for comparability with the other algorithms) only pairwise merges of components are considered: i.e. the option of deleting components entirely is eschewed. Finally panel (d) shows the result of applying the $B(i, j)$ criterion. It will be noted that the WilliamsMaybeck reduction preserves the shape of the 50\% mixture contour well (except for the loss of its secondary peak), but creates the greatest distortion of the $95 \%$ contour. In contrast, the reduction using $B(i, j)$ is the best at preserving the shape of the $95 \%$ contour, but causes greater distortion to the $50 \%$ contour. This doubtless reflects the fact that the Williams-Maybeck criterion is aiming to minimise absolute differences of the reduced pdf from the original, where the Kullback-Leibler bound aims to avoid large ratio reductions. The behaviour of Salmond's criterion is intermediate between the others, though interestingly it is the only one to preserve the secondary peak in the $50 \%$ contour (although shifting it somewhat to the north).

Fig. 3 considers the Kullback-Leibler discrimination of the reduced mixture with respect to the original mixture, and

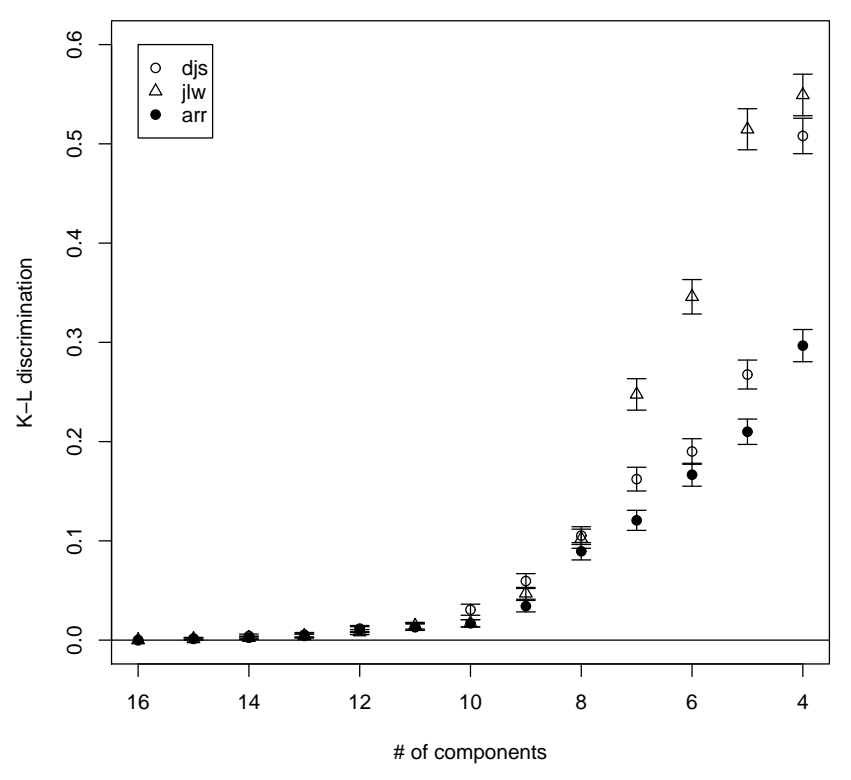

Fig. 3. Kullback-Leibler discrimination of the reduced mixture with respect to the original mixture.

shows how it evolves as the merging process progresses. Results for Salmond's criterion are shown as hollow circles, for the Williams-Maybeck measure as triangles, and for the $B(i, j)$ criterion as solid disks. The $\mathrm{K}-\mathrm{L}$ discrimination was calculated using Monte Carlo integration using 100,000 points drawn from the original mixture; to improve comparability, the same points were used for each integration. The figure includes $\pm 2 \sigma$ tolerance bounds for each plotted point.

From the figure, it is evident that there is very little difference in the performance of the algorithms as they reduce the mixture down to 11 components. At this stage, the K$\mathrm{L}$ discrimination is about 0.013 for each algorithm, which means that it will require about 350 samples from the 'true' mixture to distinguish it from the reduced one with an expected $\log$-likelihood-ratio of $\log 100$. As the number of components is further reduced down to four, the discrimination increases more rapidly, reaching 0.51 for the Salmond criterion, 0.55 for the Williams-Maybeck criterion, and 0.30 for the $B(i, j)$ criterion, corresponding to sample sizes of 9,8 and 15 respectively.

\section{CONCLUSION}

This paper has examined two algorithms proposed in the literature for reducing a Gaussian mixture to a mixture with fewer components, namely those due to Salmond [1] and to Williams and Maybeck [2], [3]. An element of both of these algorithms is successively to merge pairs of components, at each stage replacing the merged pair by a single Gaussian component with the same moments up to the second order.

It has been shown that each of these algorithms can give rise to anomalous behaviour in certain circumstances:

- Salmond's algorithm chooses for merging the pair of 


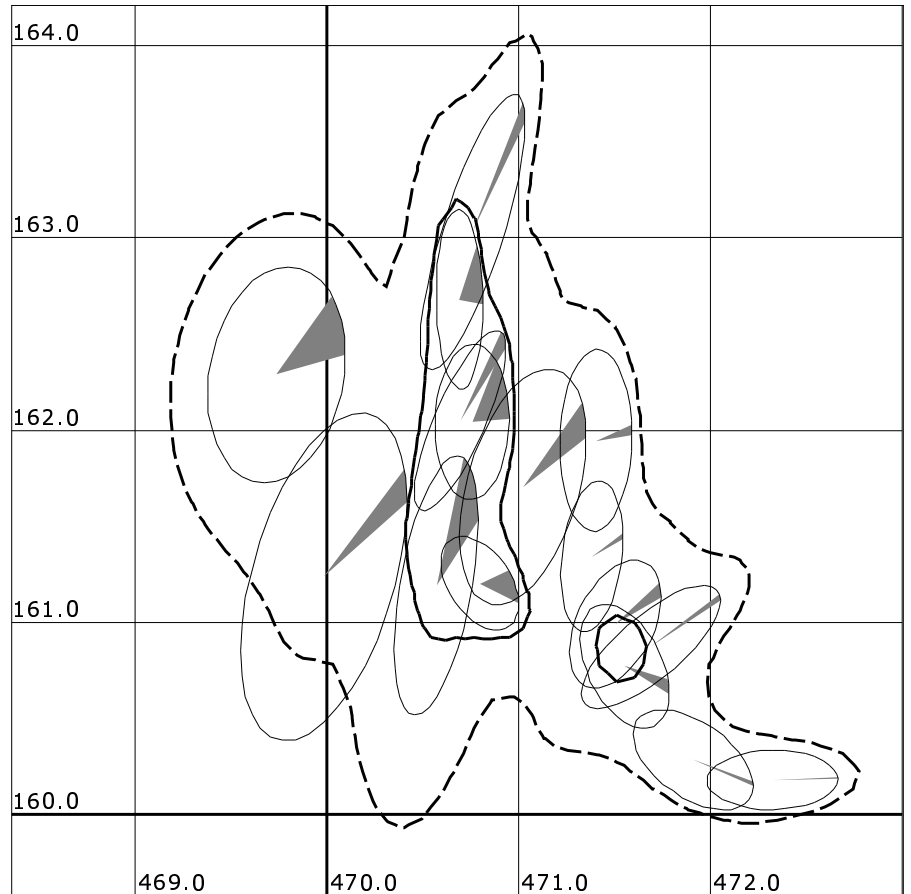

(a) Original mixture

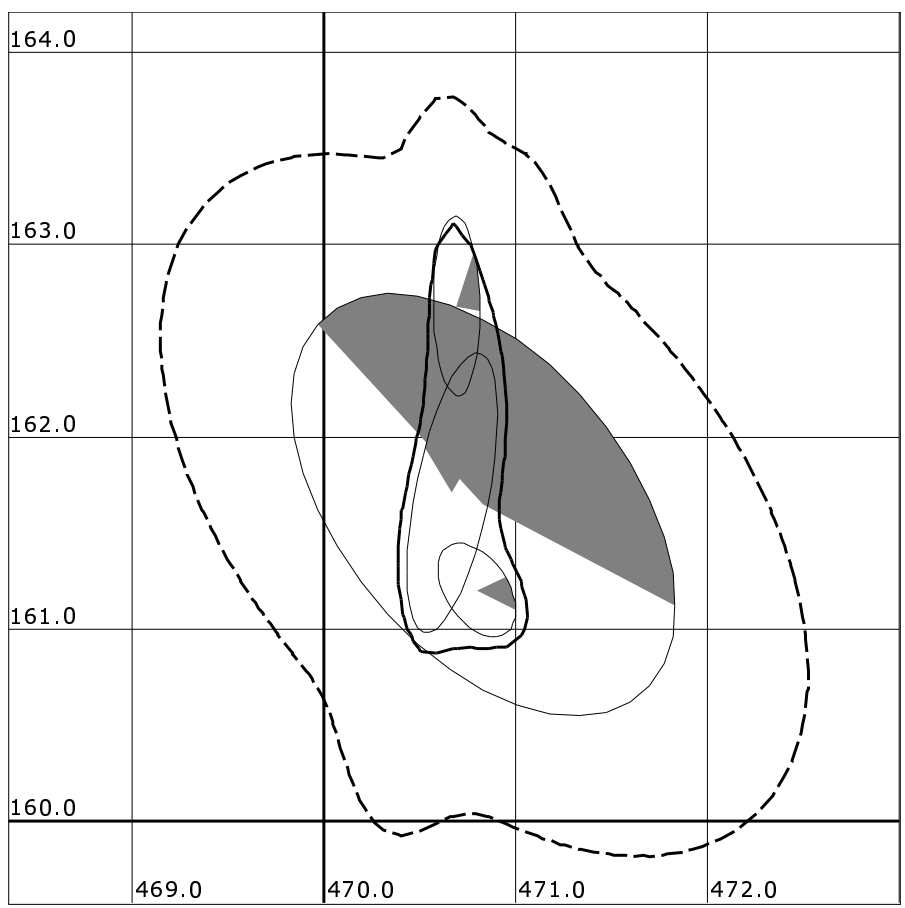

(c) Williams-Maybeck reduction

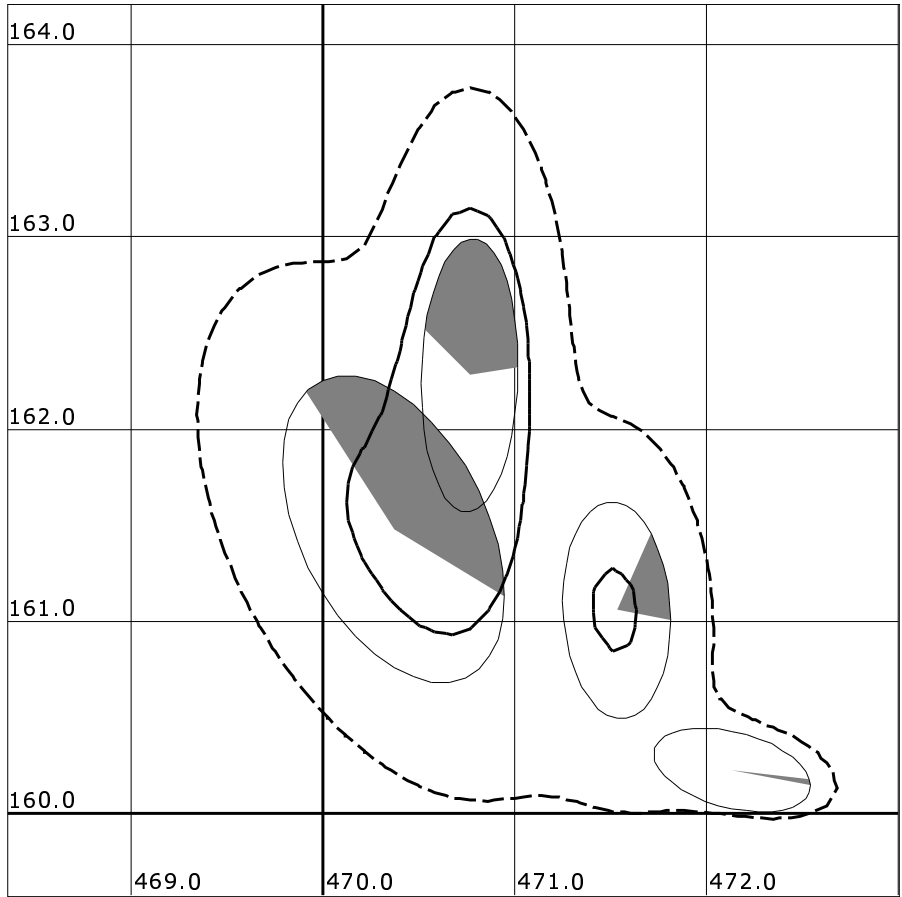

(b) Salmond reduction

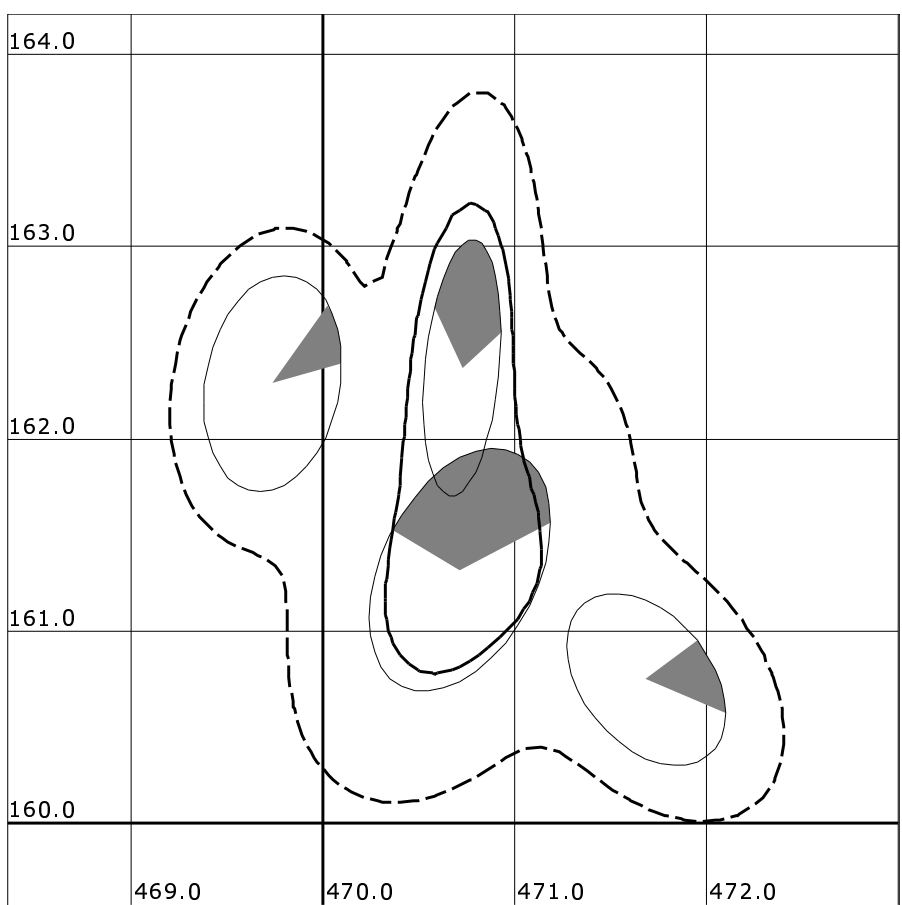

(d) K-L bound reduction

Fig. 2. Comparison of reduction algorithms applied to a 16-component mixture over 15 dimensions. 
components whose means are closest together, even if their covariance matrices are very different.

- In Salmond's algorithm, adding a new component to a mixture can alter the order in which existing components are merged, even if-indeed, especially if - the new component is far remote from the existing components and is therefore not itself a candidate for merging.

- The Williams algorithm has a tendency to select for merging a pair of components with large variances, even if their means are much further apart (in relation to their standard deviations) than another pair of components with smaller variances. This effect is particularly pronounced with state vectors of high dimension.

The paper went on to propose a new algorithm, again based on pairwise merging of components, but in which the choice of components for merging is based on an easily-computed upper bound of the Kullback-Leibler discrimination of the post-merge mixture with respect to the pre-merge mixture, as defined in (21). It has been shown that this criterion avoids the anomalies described above.

An indicative example has been presented, using a dataset derived from terrain-referenced navigation, in which it is required to reduce a 16-component mixture over 15 dimensions down to four components. The final mixture arrived at by each of the three algorithms has been illustrated, along with data on the Kullback-Leibler discrimination of the reduced mixture with respect to the original mixture, showing how this grows as the reduction process proceeds. In the example, the final $\mathrm{K}$ L discrimination was over $30 \%$ lower using the new algorithm than with either of the others.

Further work is desirable to compare at greater length the performance of the algorithms considered in this paper within particular application scenarios. A possible approach to this would be to repeat analyses along the lines of Sec. VIII for a large sample of mixture reduction problems within the particular application area.

\section{APPENDIX}

\section{PROOFS OF THEOREMS}

(In each proof we use the standard inequality $\log x \leq x-1$.)

\section{Theorem 3.2}

Proof: Using (5) it is straightforward to show that

$$
\begin{aligned}
2 d_{\mathrm{kl}}(f, & (1, \boldsymbol{\mu}, P)) \\
= & 2 \int_{\Re^{d}} f(\mathbf{x}) \log f(\mathbf{x}) \mathrm{d} \mathbf{x}+d \log 2 \pi \\
& +\log \operatorname{det} P+\int_{\Re^{d}}(\mathbf{x}-\boldsymbol{\mu})^{T} P^{-1}(\mathbf{x}-\boldsymbol{\mu}) f(\mathbf{x}) \mathrm{d} \mathbf{x} \\
= & 2 \int_{\Re^{d}} f(\mathbf{x}) \log f(\mathbf{x}) \mathrm{d} \mathbf{x}+d \log 2 \pi \\
& +\log \operatorname{det} P+\operatorname{tr}\left[P^{-1} \int_{\Re^{d}}(\mathbf{x}-\boldsymbol{\mu})(\mathbf{x}-\boldsymbol{\mu})^{T} f(\mathbf{x}) \mathrm{d} \mathbf{x}\right]
\end{aligned}
$$

where we have used the standard identity $\mathbf{v}^{T} M \mathbf{v}=\operatorname{tr}\left(M \mathbf{v} \mathbf{v}^{T}\right.$ ) (cf. [10, p. 34]). Now, writing $\Delta \boldsymbol{\mu}=\boldsymbol{\mu}-\boldsymbol{\mu}_{*}$ we have

$$
\int_{\Re d}(\mathbf{x}-\boldsymbol{\mu})(\mathbf{x}-\boldsymbol{\mu})^{T} f(\mathbf{x}) \mathrm{d} \mathbf{x}=P_{*}+\Delta \boldsymbol{\mu} \Delta \boldsymbol{\mu}^{T}
$$

Consequently, if $\lambda_{1}, \ldots \lambda_{d}$ are the eigenvalues of $P^{-1} P_{*}$, we have:

$$
\begin{aligned}
2\left[d_{\mathrm{kl}}(\right. & \left.f,(1, \boldsymbol{\mu}, P))-d_{\mathrm{kl}}\left(f,\left(1, \boldsymbol{\mu}_{*}, P_{*}\right)\right)\right] \\
=\quad & \log \operatorname{det} P-\log \operatorname{det} P_{*} \\
& \quad+\operatorname{tr}\left[P^{-1}\left(P_{*}+\Delta \boldsymbol{\mu} \Delta \boldsymbol{\mu}^{T}\right)\right]-\operatorname{tr}\left[P_{*}^{-1} P_{*}\right] \\
= & -\log \operatorname{det}\left(P^{-1} P_{*}\right) \\
& \quad+\operatorname{tr}\left(P^{-1} P_{*}\right)+\operatorname{tr}\left(P^{-1} \Delta \boldsymbol{\mu} \Delta \boldsymbol{\mu}^{T}\right)-d \\
= & -\log \prod_{i=1}^{d} \lambda_{i}+\sum_{i=1}^{d} \lambda_{i}-d+\operatorname{tr}\left(P^{-1} \Delta \boldsymbol{\mu} \Delta \boldsymbol{\mu}^{T}\right) \\
= & \sum_{i=1}^{d}\left(-\log \lambda_{i}+\lambda_{i}-1\right)+\operatorname{tr}\left(P^{-1} \Delta \boldsymbol{\mu} \Delta \boldsymbol{\mu}^{T}\right) \\
\geq & 0
\end{aligned}
$$

with equality only if $\Delta \boldsymbol{\mu}=\mathbf{0}$ and $\lambda_{i}=1$ for $i=1, \ldots d$, i.e. if $P^{-1} P_{*}=I$. This proves the theorem.

\section{Theorem 3.4} have:

Proof: The proof is similar to that of [9, Theorem 4.3.3]. We

$$
\begin{aligned}
w d_{\mathrm{kl}}\left(f_{1}, f_{2}\right)-d_{\mathrm{kl}}\left(w f_{1}+\bar{w} h, w f_{2}+\bar{w} h\right) \\
=\quad w \int_{\Re^{d}} f_{1}(\mathbf{x}) \log \frac{f_{1}(\mathbf{x})}{f_{2}(\mathbf{x})} \mathrm{d} \mathbf{x} \\
\quad-\int_{\Re^{d}}\left(w f_{1}(\mathbf{x})+\bar{w} h(\mathbf{x})\right) \log \frac{w f_{1}(\mathbf{x})+\bar{w} h(\mathbf{x})}{w f_{2}(\mathbf{x})+\bar{w} h(\mathbf{x})} \mathrm{d} \mathbf{x} \\
=\quad \int_{\Re^{d}} w f_{1}(\mathbf{x}) \log \frac{f_{1}(\mathbf{x})\left[w f_{2}(\mathbf{x})+\bar{w} h(\mathbf{x})\right]}{f_{2}(\mathbf{x})\left[w f_{1}(\mathbf{x})+\bar{w} h(\mathbf{x})\right]} \mathrm{d} \mathbf{x} \\
\quad+\int_{\Re^{d}} \bar{w} h(\mathbf{x}) \log \frac{w f_{2}(\mathbf{x})+\bar{w} h(\mathbf{x})}{w f_{1}(\mathbf{x})+\bar{w} h(\mathbf{x})} \mathrm{d} \mathbf{x} \\
\geq \quad \int_{\Re^{d}} w f_{1}(\mathbf{x})\left[1-\frac{f_{2}(\mathbf{x})\left[w f_{1}(\mathbf{x})+\bar{w} h(\mathbf{x})\right]}{f_{1}(\mathbf{x})\left[w f_{2}(\mathbf{x})+\bar{w} h(\mathbf{x})\right]}\right] \mathrm{d} \mathbf{x} \\
\quad+\int_{\Re^{d}} \bar{w} h(\mathbf{x})\left[1-\frac{w f_{1}(\mathbf{x})+\bar{w} h(\mathbf{x})}{\left.w f_{2}(\mathbf{x})+\bar{w} h(\mathbf{x})\right] \mathrm{d} \mathbf{x}}\right. \\
=\quad 1-\int_{\Re^{d}}\left(w f_{2}(\mathbf{x})+\bar{w} h(\mathbf{x})\right) \frac{w f_{1}(\mathbf{x})+\bar{w} h(\mathbf{x})}{w f_{2}(\mathbf{x})+\bar{w} h(\mathbf{x})} \mathrm{d} \mathbf{x} \\
=\quad 0
\end{aligned}
$$

\section{ACKNOWLEDGMENT}

This work was partly supported by QinetiQ Ltd, Farnborough, UK, under funding from the Applied Research Programme of the UK Ministry of Defence. The author would like to thank David Salmond for helpful comments on a draft of this paper.

\section{REFERENCES}

[1] D. J. Salmond, "Mixture reduction algorithms for target tracking in clutter," in Signal and Data Processing of Small Targets 1990, 1990, pp. 434-45, proceedings of SPIE Vol. 1305.

[2] J. L. Williams, "Gaussian mixture reduction for tracking multiple maneuvering targets in clutter," Master's thesis, M.S.E.E. Thesis, Air Force Institute of Technology, Wright-Patterson Air Force Base, OH, 2003.

[3] J. L. Williams and P. S. Maybeck, "Cost-function-based Gaussian mixture reduction," in Sixth International Conference on Information Fusion. ISIF, 2003.

[4] S. Blackman and R. Popoli, Design and Analysis of Modern Tracking Systems. Artech House, 1999. 
[5] P. D. Groves, R. J. Handley, and A. R. Runnalls, "Optimising the integration of terrain-referenced navigation with INS and GPS," in Proceedings of Institute of Navigation National GNSS2004, Long Beach, California, USA, September 2004, pp. 1048-59.

[6] A. R. Runnalls, P. D. Groves, and R. J. Handley, "Terrain-referenced navigation using the IGMAP data fusion algorithm," in 61st Annual Meeting of the Institute of Navigation, Institute of Navigation, 3975 University Drive, Fairfax, VA 22030, www. ion.org, June 2005, pp. 976-87.

[7] S. Kullback and R. A. Leibler, "On information and sufficiency," Annals of Mathematical Statistics, vol. 22, pp. 79-86, 1951.

[8] T. M. Cover and J. A. Thomas, Elements of Information Theory. Wiley, 1991.

[9] R. E. Blahut, Principles and Practice of Information Theory. AddisonWesley, 1987.

[10] C. R. Rao, Linear Statistical Inference and its Applications, 2nd ed. Wiley, 1973. 
[5] P. D. Groves, R. J. Handley, and A. R. Runnalls, "Optimising the integration of terrain-referenced navigation with INS and GPS," in Proceedings of Institute of Navigation National GNSS2004, Long Beach, California, USA, September 2004, pp. 1048-59.

[6] A. R. Runnalls, P. D. Groves, and R. J. Handley, "Terrain-referenced navigation using the IGMAP data fusion algorithm," in 61st Annual Meeting of the Institute of Navigation, Institute of Navigation, 3975 University Drive, Fairfax, VA 22030, www. ion.org, June 2005, pp. 976-87.

[7] S. Kullback and R. A. Leibler, "On information and sufficiency," Annals of Mathematical Statistics, vol. 22, pp. 79-86, 1951.

[8] T. M. Cover and J. A. Thomas, Elements of Information Theory. Wiley, 1991.

[9] R. E. Blahut, Principles and Practice of Information Theory. AddisonWesley, 1987.

[10] C. R. Rao, Linear Statistical Inference and its Applications, 2nd ed. Wiley, 1973. 